\title{
EXOTIC MATTER RESEARCH IN SPACE
}

$\operatorname{Rom} 2 \mathrm{~F} / 2001 / 06$

\author{
P.PICOZZA, A. MORSELLI \\ Dept. of Physics, Univ. of Roma "Tor Vergata" and INFN, Sezione di Roma 2, Roma, \\ Via della Ricerca Scientifica 1, 00133, Italy \\ E-mail: picozza@roma2.infn.it,morselli@roma2.infn.it
}

\begin{abstract}
The direct detection of annihilation products in cosmic rays offers an alternative way to search for dark matter particles candidates. Here we will see in particular that the study of the spectrum of antiproton and positrons offers good possibilities to perform this search and we will review our experimental effort in this direction.
\end{abstract}

\section{Probing for dark matter with cosmic rays space experiments}

Galactic dark matter was suggested to solve the discrepancy between observed (luminous) matter in the Universe and that inferred from dynamical considerations. A matter density of about $30-40 \%$ of the critical density of the Universe can be composed of dark matter. One possible form of dark matter could be weakly interacting massive particles (WIMPs) and a good candidate for WIMP's is the Lightest Supersymmetric Particle (LSP) in R-parity conserving SUSY models. In most of the Supersymmetric theories the LSP is the neutralino $\chi$ that is a combination of the partners of the $\gamma, Z$ and the neutral Higgs particles (see $\square$ and references threin). Neutralinos are Majorana fermions and will annihilate with each other in the halo producing leptons, quarks, gluons, gauge bosons and Higgs bosons. The quarks, gauge bosons and Higgs bosons will decay and/or form jets that will give rise to antiprotons (and antineutrons which decay shortly to antiprotons).

The idea of exploiting cosmic antiprotons measurements to probe pnconventional particle physics and astrophysics scenarios has a long history 2 and was stimulated by early reports 3 of unexpectedly large values for the antiproton to proton ratio in cosmic rays.

Quantitative estimation of the antiproton flux due to dark-matter annihilation requires assumptions about the WIMP mass and dark matter density.

The solar modulation introduce incertanties in the antiproton spectrum at low energies (below $1 \mathrm{GeV}$ ), so the best place to look from a deviation of the spectra is at high energies where there can be a neutralino signal rather sharply peaked at an energy higher than the maximum in the background if its spectrum decreases rapidly at low energies. This effect can be produced by high mass neutralinos with negligible branching ratio into $b \bar{b}$ or $t \bar{t}$, which is the case e.g. for a very pure heavy Higgsino-like neutralino.

In figure 1 (on the right) there are the experimental data for the antiproton flux 5 together with the distortion on the antiproton flux (dashed line) due to one possible contribution from neutralino annihilation (dotted line, from 6 ). Total expected flux is shown by solid line. The antiproton data that PAMELA would obtain in a single year of observation for one of the Higgsino annihilation models are shown by grey circles. The PAMELA experiment will be described in the next section.

The Ninth Marcel Grossmann Meeting, Rome, July 2 - 8, 2000 

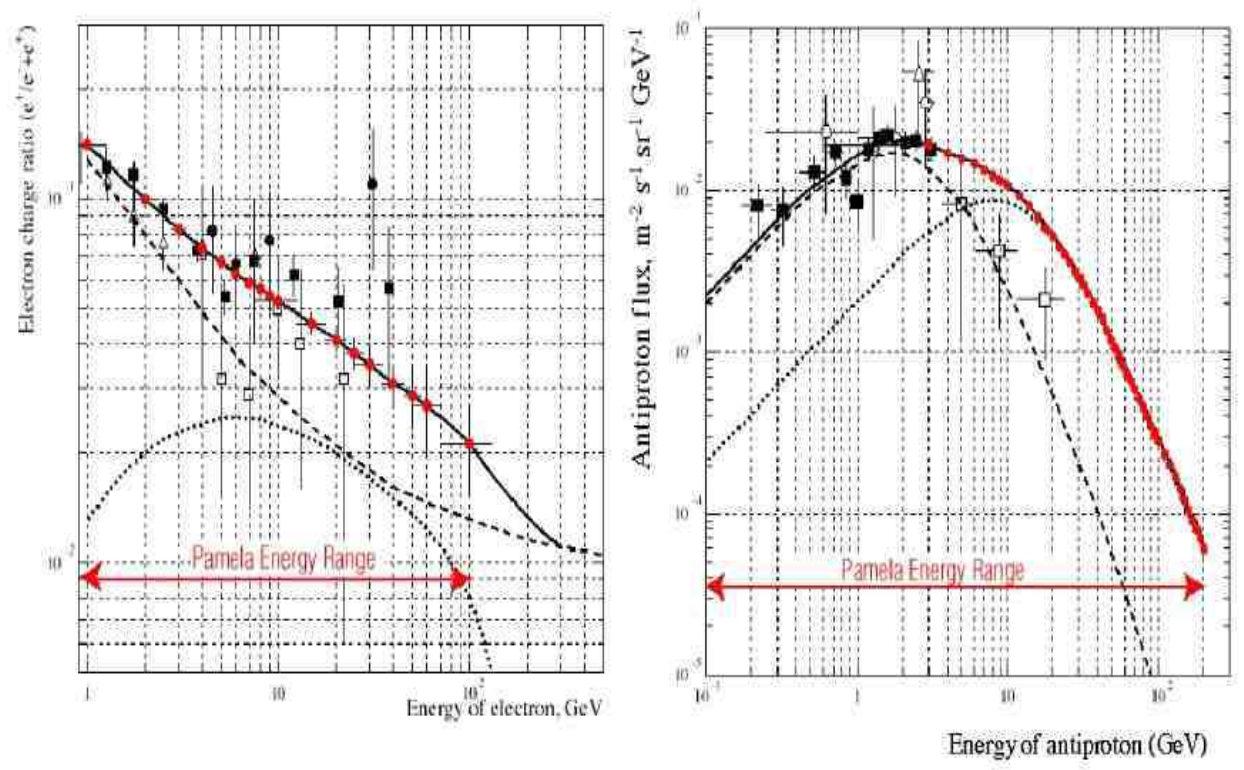

Figure 1. Distortion of the secondary positron fraction (on the left) and secondary antiproton flux (on the right) induced by a signal from a heavy neutralino

Exotic dark matter can also be investigated with positrons. Electrons are a primary component of cosmic rays and emerge from ambient material in an acceleration process that may or may not be identical to that which generates energetic nucleons. Positrons are thought to be mostly secondary in origin. WIMPs could contribute to the positron flux by direct annihilation into $\mathrm{e}^{+} \mathrm{e}^{-}$, and to continuum positrons from the other annihilation channels 6 . This will be seen as a excess or bump beginning at a few $\mathrm{GeV}$ and extending upward in energy to a point depending on the WIMP mass. Current experimental data is not sufficient to make solid conclusions, but there is some evidence for a positron excess $\mathrm{Q}$.

In figure 1 (on the left) there are the experimental data 0 for the positron fraction together with the distortion of the secondary positron fraction (dashed line) que to one possible contribution from neutralino annihilation (dotted line, from 8 ). The expected data from the PAMELA experiment in the annihilation scenario for one year of operation are shown by grey circles.

This effort will be complementary to a similar search with high-energy gammaray instruments such as GLAST $(10-300 \mathrm{GeV})$ and ground-based air Cherenkov telescopes (100-1000 GeV) looking of possible signature of the existence of the LSP as a bump in the spectrum of the diffuse gamma ray background around the neutralino mass due to neutralino annihilation in the halo 1 


\section{2 the PAMELA apparatus}

PAMELA is an satellite-borne magnet spectrometer built by the WiZard-PAMELA collaboration 0 . It will be installed on-board of the RESURS-5 ARTIKA satellite to be launched in the 2003 for a mission at least three years long. The satellite orbit is polar, sun-synchronous and $700 \mathrm{~km}$ high.

The list of the people and the Institution involved in the collaboration together with the on-line status of the project is available at http://wizard.roma2.infn.ith. The Pamela telescope, shown in figure 2, consists of the following elements: a magnet + tracker system, an imaging calorimeter, a Transition-Radiation-Counter (TRD), scintillation counter hodoscopes for Time-of-Flight and Trigger, an anticoincidence scintillation counter. The magnet + tracker system consist of 5 permanent magnets, each $8 \mathrm{~cm}$ high, interleaving 6 detection planes of the silicon microstrip tracker. The whole closed in a ferromagnetic screen and surrounded on its sides by a system of anticoincidence scintillation counters. The resolution of the tracking system is about $4 \mu \mathrm{m}$. The magnetic field inside the magnet will be $\sim 0.4 \mathrm{~T}$, so the Maximum Detectable Rigidity (MDR) will be around $800 \mathrm{GV} / \mathrm{c}$.

\subsection{PAMELA Scientific Objectives}

The observational objectives of the PAMELA instrument are the measurement of the spectra of antiprotons, positrons and nuclei in a wide range of energies, the search for primordial antimatter and the study the cosmic ray fluxes over half a solar cycle. Data gathered with the PAMELA instrument will deal with a wide range of fundamental issues. These include:

- the role of Grand Unified Theories in Cosmology in relation to antimatter and dark matter.

- the understanding of the acceleration and propagation of cosmic rays.

- the role of solar, terrestrial and heliosperic relationships to energetic particle propagation in the heliosphere.

The PAMELA observations will extend the results of balloon-borne experiments over an unexplored range of energies with unprecedented statistics and will complement information gathered from Great Space Observatories. These observational objectives can be schematically listed in the following points:

- Measurement of the energy antiproton spectrum in a large energy range: from $100 \mathrm{MeV}$ up to $150 \mathrm{GeV}$ (present limits $0.4-20 \mathrm{GeV}$ );

- Measurement of the energy positron spectrum in a large energy range: from 100 $\mathrm{MeV}$ up to $200 \mathrm{GeV}$ (present limits $0.7-30 \mathrm{GeV}$ );

- Search for anti-nuclei with a sensitivity of $610^{-8}$ in the anti-helium/helium ratio (present limit about $10^{-5}$ );

- Measurement of the electron energy spectrum up to $1000 \mathrm{GeV}$;

- Continuous monitoring of the cosmic rays solar modulation during and after the 23rd maximum of solar activity;

- Studies of the time and energy distributions of the energetic particles emitted in solar flares. 


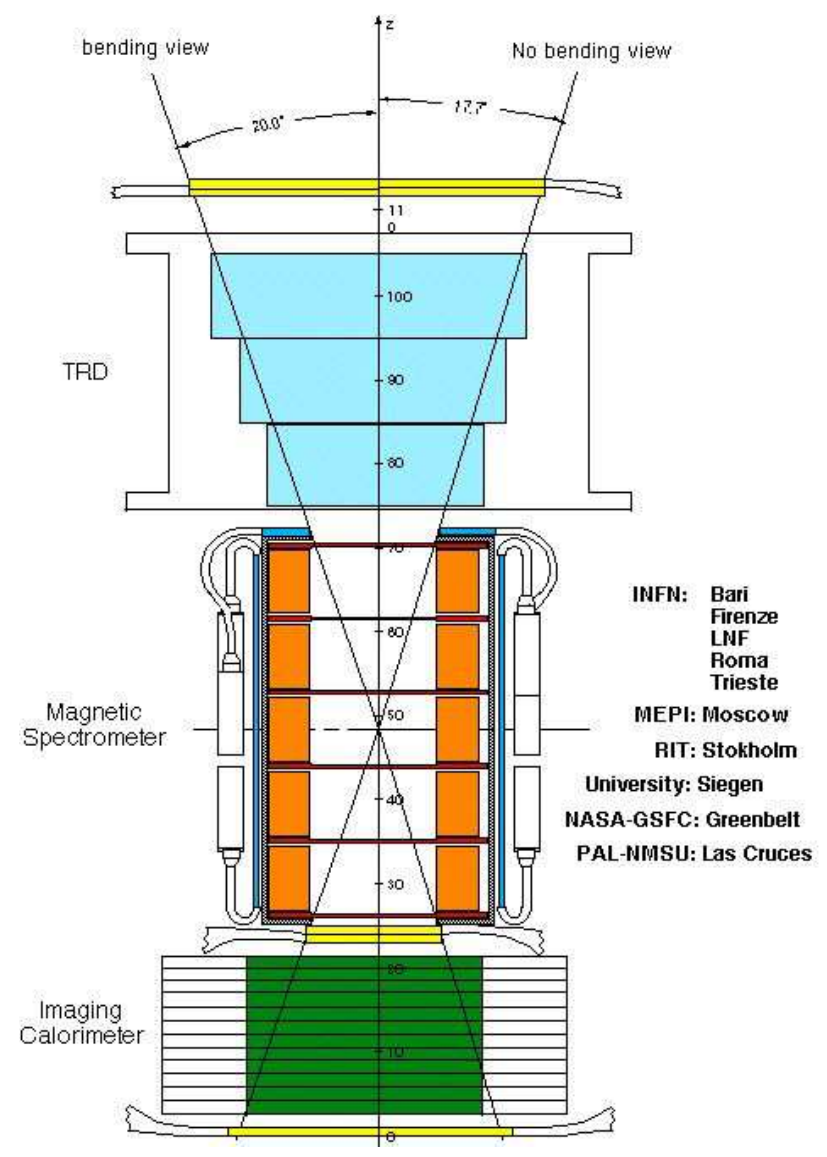

Figure 2. Schematic of the PAMELA baseline instrument.

The low energy antiproton and positron measurements and the last two objectives are peculiar of the PAMELA experiment because the satellite travels in a polar orbit. It spends a large fraction of its time in the high latitude and Polar Regions, where the cut-off due to the terrestrial magnetic field is negligible.

The scientific relevance of these objectives is enhanced by the length of the mission, that is planned to last not less than three years, but could be prolonged for many other years because of the orbit altitude and the maximization of the electric power due to its sun-synchronism.

In the following sub-sections the above objectives are discussed in some detail.

- Antiprotons

Antiprotons have been observed in the cosmic rays since 1979 by balloon-borne experiments; prior to these measurements it was generally expected that all primary cosmic rays experienced the same basic history during their acceleration and propagation. Similarly it was assumed that all secondary were produced and stored in the same regions of the Galaxy. These assumptions were quite adequate to explain 
the observations of secondary $\mathrm{Z} \geq 3$ nuclei in cosmic rays.

Because of atmospheric backgrounds and limited flight times, balloon-borne experiments can only measure the energy spectrum of antiprotons up to about $20 \mathrm{GeV}$. In addition, the sensitivity of the balloon observations is limited by the difficulty in eliminating large fluxes of atmospheric secondary particles. PAMELA will be able to measure the energy spectrum of antiprotons up to $150 \mathrm{GeV}$.

- Positrons and Electrons.

Positrons and electrons are unique among cosmic rays because they are the lightest charged leptons. Due to their low mass, high-energy electrons and positrons undergo interactions with the ISM, which result in severe energy losses at high energies. While most of the observed electrons are believed to be of primary origin, the origin of positrons is yet to be established. Positrons are even harder to observe than antiprotons due to the high flux of protons (more than 1000 times higher). All observations to date have suffered from the risk of subtracting significant background. The majority of data shows an excess of positrons above the flux expected by the simple leaky-box model and may even indicate a rise in the positron/electron ratio at energies greater than $15 \mathrm{GeV}$. These direct observations, combined with the observation of a positron annihilation emission line from the galactic disk and the high antiproton fluxes, give rise to questions such as:

1. Are there positrons in the cosmic rays that are not produced as secondary?

2. Is there a relationship between the antiproton and positron excesses?

3. If positrons are indeed all secondary particles, at what point do the radiative losses become important?

Observation of positron over a very large energy range should yield new insights into galactic processes. In particular, as we showed, the signature of WIMP particle existence in Dark Matter could be found in the high-energy spectrum of positron. As for antiprotons, PAMELA will aim to measure accurately the spectra of positrons from low (cut-off) energies up to the highest energies attainable (about $200 \mathrm{GeV}$ ). Furthermore, together with the measurement of the spectra of electrons (up to about $1000 \mathrm{GeV}$ ) PAMELA can provide information on the:

1. Acceleration of electrons and the distribution of acceleration sites;

2. Cosmic-ray lifetime, and the physical conditions in the containment volume;

3. Magnitude of re-acceleration by interstellar shock waves.

- Search for antimatter.

Detection of antimatter of primary origin in cosmic rays would be a discovery of fundamental significance. Cosmic-ray searches that have been made so far have yielded only upper limits of one part in $10^{-4}$ for heavy nuclei $(\mathrm{Z}>2)$ and one part in $10^{-5}$ for helium 12. The detection of anti-nuclei in cosmic rays would provide direct evidence of the existence of antimatter in the universe. Baryons and photons were produced in the Big Bang in equal amount, but from observation of the $2.7 \mathrm{~K}$ cosmic background radiation and the present matter density of the universe we know that only about one baryon remains for ever $10^{9}$ photons. The current theory suggests that the remaining matter is the remnant of the almost complete annihilation of matter and antimatter at some early epoch, which stopped only when there was 
no more antimatter to annihilate. Starting from a matter/antimatter symmetric Universe, the required conditions for a following asymmetric evolution are the CP violation, the baryon number non-conservation and a non equilibrium environment. On the basis of gamma-ray observations, the coexistence of condensed matter and antimatter on scales smaller than that of clusters of galaxies has been virtually ruled out. However, no observations presently exclude the possibility that the domain size for establishing the sign of $\mathrm{CP}$ violation is as large as a cluster or super-cluster of galaxies. For example, there could be equality in the number of super-clusters and anti-super-clusters. Similarly, there is nothing that excludes the possibility that a small fraction of the cosmic rays observed at Earth reach our Galaxy from nearby super-clusters. PAMELA will search for anti-nuclei with sensitivity on the anti-helium/helium ratio of some units in $10^{-8}$.

-Additional objectives

The continuous determination of the direction, latitude and longitude of the primary electrons, positrons, protons, antiprotons and light nuclei during several years and over a large energy range (100 $\mathrm{MeV}$ up to several tens $\mathrm{GeV}$ ) will provide a great opportunity to investigate other scientific issues. The PAMELA experiment will be able to address these additional objectives besides the primary ones above described. Indeed, during its orbiting around the Earth, the satellite will encounter all that kind of events that are related to the solar activity and to the terrestrial geomagnetic effects.

The analysis of all data gathered over its mission will provide a significant complement to the measurements performed so far with dedicated experiments of different concepts. The additional objectives of PAMELA are the following:

1. Modulation of galactic cosmic rays in the heliosphere;

2. Solar flare particle spectra;

3. Distribution and acceleration of solar cosmic rays (SCR's) in the internal heliosphere;

4. Magnetosphere and magnetic field of the Earth;

5. Stationary and disturbed fluxes of high energy particles in the Earth's magnetosphere;

6. Anomalous component of cosmic rays.

The PAMELA mission will carry out its observations during and after the maximum of the 23rd solar activity cycle. The modulation effect on electrons, positrons, protons and nuclei during this period will be investigated in order to find any dependence on charge sign, energy (rigidity) and mass of the particles. Latitude and longitude distributions of the observed fluxes will be analysed as a function of the solar activity to look for possible correlation.

Most of the satellite measurements on the composition and spectrum of solar energetic particles from the flares are limited to energies below a few tens $\mathrm{gf} \mathrm{MeV}$. Experiments carried by GOES-7, SAMPEX and NINA $1 \& 2$ telescope 13 , could determine the spectrum of heavy nuclei to about $100 \mathrm{MeV} / \mathrm{n}$ for a few flares. Using PAMELA it is possible to measure the energy spectrum of nuclei from helium to at least oxygen from about $100 \mathrm{MeV} / \mathrm{n}$ up to an energy where the solar energetic particles can be distinguished from the galactic cosmic rays. Solar flares with 
generation of similar particles are rare but, as of 2002, when the solar activity is at its maximum, about ten of such events per year will be available. The composition and the spectral shape of nuclear components could vary from flare to flare and it would be very exciting to relate these variations to the physical conditions in the flare sites.

For the first time, there will be an opportunity to observe at the same time high energy solar particles with different charges and different masses. The observation of such particles will not only allow to choose between different SCR's acceleration processes on the Sun, but also to gather information on the distribution and acceleration processes of the particles in the internal part of the heliosphere. The observation of SCR's, the determination of energy and temporal distributions of various components will allow to carry out the internal tomography of the heliosphere: the shock wave propagation and the fluxes of solar wind drivers (coronal mass ejection) will be investigated.

The experimental data obtained by PAMELA both in conditions of quiet and disturbed Earth's magnetosphere will allow to study the variations of current systems and their influence on the trajectory of cosmic rays and on the geomagnetic rigidity thresholds.

The altitude of the PAMELA orbit is about $700 \mathrm{~km}$. For almost one third of its orbits, the instrument will cross the internal part of the Earth radiation belt (the so called Brazilian anomaly region) giving the opportunity of observing both primary cosmic rays and the high energy particles of the Earth radiation belt itself.

The anomalous component of cosmic rays consists of partially (or single) ionised interstellar neutral atoms accelerated at the termination shock and penetrating inside the heliosphere. Their energy can vary from $10 \mathrm{MeV}$ up to several hundred $\mathrm{MeV}$ which is sufficient to reach the vicinity of the Earth and to be observed by satellites at high latitudes or outside the magnetosphere. Due to the inclination of the PAMELA's satellite orbit (98 degrees) there is an opportunity to carry out measurements of the anomalous component of cosmic rays both at high latitudes and in the radiation belt of the Earth.

\subsection{The PAMELA telescope.}

The PAMELA telescope design is well defined and completed. In the past two years prototypes of detectors and sub-systems of the telescope have been built and tested, measuring their performances. Therefore, the expected behaviour of the whole PAMELA experiment is well known and the required techniques already defined and tested.

The concept of the PAMELA telescope is the same on which the proposed WIZARD experiment was based:

1. A magnetic spectrometer to determine the sign of the electric charge with a very high confidence degree, and to measure the momentum of the particles up to the highest energies for which a useful flux of rare particles (as antiprotons and positrons) can be collected;

2. An imaging calorimeter that can give, besides the measurement of the energy released by the interacting particle (and indeed of the extra energy released in 
an annihilation event), the pattern of the interaction of the particle inside the calorimeter, in order to identify the particle itself. This last point, observing the annihilation pattern of antiprotons and possible anti-nuclei, allows confirmation of their identity on an "event by event" basis;

3. A velocity measurement system to help the calorimeter in the identification of the nature of the particle;

The technical choices had to be made taking into account the mass and power limits of the payload, dictated by the launch opportunity. They are the following:

Permanent magnet system and micro-strip silicon sensors for the magnetic spectrometer. The magnetic system is composed by five permanent magnet of Nd-Fe-B, each $8 \mathrm{~cm}$ high, that provide a field inside the tracking volume of about $0.4 \mathrm{~T}$. There are six planes of silicon micro-strip detectors and the tests made in the past years on PAMELA detector prototypes resulted in a spatial resolution of $2.9 \mu \mathrm{m}$. Therefore, by considering a conservative approach, the spatial resolution requirement of the PAMELA tracker is fixed to $4 \mu \mathrm{m}$;

Silicon strip sensors for the imaging calorimeter, interleaved with tungsten plates as absorber; this choice minimizes the volume of the calorimeter, and maximizes indeed its geometrical acceptance; the high granularity assumed in PAMELA allows a very good separation between electromagnetic showers and interacting or not interacting hadrons; the chosen depth of 16 radiation length allows a good resolution in the measurement of the energy of electromagnetic particles, further extending the energy spectrum measurements of electrons and positrons;

A Transition Radiation Detector (TRD) for distinguishing electromagnetic particles from hadrons up to very high energy (about $1000 \mathrm{GeV}$ ); the TRD is based on small diameter straw tubes arranged in double layer planes interleaved by carbon fibre radiator. The use of the straw tubes allows to perform many energy loss measurements along the particle trajectory bringing the selection capability of the instrument down to less than $1 \mathrm{GeV}$, and to track all particles before their entrance in the magnetic spectrometer, cleaning the sample of the particles accepted at its entrance;

Several (6) scintillation counter hodoscopes (each $7 \mathrm{~mm}$ thick) for the construction of the triggers and for the TOF measurements; the use of several hodoscopes allows independent TOF measurements, improving the precision and the safety;

Finally a set of scintillation counters covering the top edge and the sides of the magnetic spectrometer and the bottom part of the calorimeter completes the telescope, for a further labelling of contaminating events.

The main characteristics of the PAMELA experiment are:

1. A Maximum Detectable Rigidity (MDR) of $800 \mathrm{GV} / \mathrm{c}$;

2. An acceptance (geometrical factor) of $20.5 \mathrm{~cm}^{2} \mathrm{sr}$;

3. A maximum energy reachable in the antiproton spectrum measurement of 150 $\mathrm{GeV}$ or greater, depending on the content of antiprotons in cosmic rays;

4. A maximum energy reachable in the electron and positron spectrum measurement respectively of 1000 and $200 \mathrm{GeV}$;

5. A sensitivity for the anti-helium search of $610^{-8}$ in the anti-helium/helium ratio;

6. A total volume of $90 \times 90 \times 110 \mathrm{~cm}^{3}$; 
7. A total mass of $395 \mathrm{~kg}$;

8. A number of read-out channels of 43498 ;

9. A power consumption of $267 \mathrm{~W}$.

\subsection{The PAMELA rates and data flow.}

The PAMELA experiment will be put in polar orbit; therefore the total number of collected events will be high in spite of the small Geometric Factor (GF): the magnetic cut-off due to the Earth magnetic field nearly cancels on the poles, while at medium and low latitudes the bulk of cosmic rays is cut out by the Earth magnetic field, that acts for them like a mirror.

The total rate of particle collected by the PAMELA telescope, averaged on the polar orbit and on the solar activity cycle, is 3.3 event/s, i.e. about $310^{5}$ event/day (mainly protons), ranging from 2.3 event/s to 4.2 event/s going from the period of maximum solar activity to its minimum. Averaging on the solar activity of the first three years foreseen for the PAMELA flight (2002-2005) we expect to collect in $10^{8}$ $\mathrm{s}$ the following approximate numbers for particles, antiparticles and some nuclei:

\begin{tabular}{|ll|ll|}
\hline protons & $310^{8}$ & anti-protons & $310^{4}$ \\
\hline electrons & $310^{6}$ & positrons & $110^{5}$ \\
\hline He nuclei & $410^{7}$ & Be nuclei & $410^{4}$ \\
\hline C nuclei & $410^{5}$ & anti-nuclei limit & $610^{-8}$ (90\% C.L.) \\
\hline
\end{tabular}

The expected information flow from PAMELA is of the order of $2.5 \mathrm{kB} /$ trigger; with not more than $510^{5}$ trigger/day, this results in a maximum amount of information of $1.25 \mathrm{~GB} /$ day.

\subsection{The mission profile and the spacecraft RESURS-5 ARKTIKA.}

The PAMELA experiment will be installed on the up-ward side of the RESURS-5 ARKTIKA satellite, that will be continuously oriented down-ward to the Earth during all its mission, in order to fulfil a program of Earth surface observation. Furthermore the satellite will travel in a quasi-circular, about 700-km high, polar orbit. This is an optimal situation for the observation of cosmic rays:

1. the up-ward orientation of the PAMELA telescope on board of the satellite is the required direction to observe cosmic rays without interference with the Earth and keeping far away from the telescope acceptance the showers produced by quasi horizontal cosmic rays on the terrestrial atmosphere; these showers are responsible of the strong increase of the background at low zenith angles;

2. as above mentioned, the polar orbit maximizes the cosmic ray collection rate and also minimises the geomagnetic cut-off in a significant portion of the satellite trajectory (important for all scientific issues related to the solar activity and to the terrestrial geomagnetic effects);

3. finally, the large height of the orbit insures a long permanence of the satellite in space due to the very small effect of the atmosphere. The stabilisation of the

The Ninth Marcel Grossmann Meeting, Rome, July 2 - 8, 2000 
satellite is obtained by magnetic devices to make the best use of this situation without being dependent from the possible shortness of fuel.

\section{References}

1. A. Morselli, Search for Supersymmetry and Dark Matter with GLAST, $9^{\text {th }}$ Marcell Grossmann Meeting, Roma, 2000

2. J. Silk and M.Srednicki, Phys.Rev.Lett., 53, 624 (1984).

F.W. Stecker,S.Rudaz and T.F.Walsh, Phys.Rev.Lett., 35,1 (1985).

F.W. Stecker and A.J.Tylka, Astophys. Journal, 336, L51 (1989).

G. Basini, A. Morselli, M. Ricci, La Rivista del Nuovo Cimento, 12, 4 (1989). J. Silk, and A. Stebbins, 1993, ApJ 411, 439

G. Jungman and M.Kamionkowski 1995, Phys.Rev., D51, 3121.

A. Morselli The dark side of the Universe, pg.267, World Scientific Co., 1994. T. Mitsuiet al., Phys. Lett., B 389, 169 (1996).

A.Morselli, $8^{\text {th }}$ Marcel Grossmann Meeting, Jerusalem, June 1997, pg.1465

A. Bottino, F. Donato, N. Fornengo, P. Salati, 1998, Phys. Rev. D58, 123503

3. R. Golden et al., 1979, Phys. Rev. Lett. 43, 1196

Buffington, A., et al., 1981, ApJ 248, 1179

R. Golden, S.Stephens, Space Sciece Rev., 46, 31 (1987).

4. T.K. Gaisser and B.G. Mauger, Phys.Lett. , 30 , 1264 (1973).

T.K. Gaisser and R.K. Schaefer, Astrophys. Journal, 394, 174 (1992).

M. Simon and U. Heinbach, Astophys. Journal, 456, 519 (1996).

T. Mitsui et al., Phys. Lett., B 389, 169 (1996).

5. D. Bergström et al. 2000, ApJ Letters, 534, L177 and references therein.

6. P. Ullio 1999, astro-ph/9904086

7. O.Adriani et al. 1999, 24th Int. Cosmic Ray Conference, Rome, 3, 591, 1995

— 25th Int. Cosmic Ray Conference, OG.4.2.04, Salt Lake City, 1999.

— "Frontier Objects in Astrophysics and Particle Physics", F. Giovannelli and G. Mannocchi edit. , SIF, Bologna, 65, 585.

A. Morselli, P.Picozza 1999, The PAMELA apparatus for the search of antimatter in cosmic rays, Topics in Cosmic-Ray Astrophysics Editor: M. DuVernois, pg.37. Nova Science Publisher.

8. E. Baltz and J. Edsjö, 1999 Phys. Rev. D 59, 023511.

9. S. Couto et al. 1999, Astropart. Phys. 11, 429 and references therein.

10. M. Boezio et al. 2000, ApJ, 532, 653 and references therein.

11. R.L.Golden et al., Nuovo Cimento, 105 B (1990), 191

12. G.F.Smoot et al., Phys. Rev. Lett., 35 (1975) 258.

Ormes J.F. et al., 24th ICRC (Rome), 3 (1995) 92.

13. Bakaldin A., et al. , Astroparticle Physics 8, (1997), 109.

- Nuclear Instruments and Methods A424, 414, (1999)

A. Morselli, R.Sparvoli , Topics in Cosmic-Ray Astrophysics (Volume 230 in Horizons in World Physics) Editor: M. DuVernois, Nova Science Publisher 1999, 3.

R.Sparvoli et al. , Nucl. Phys. B (Proc. Suppl.), 85, 28 , 2000.

The Ninth Marcel Grossmann Meeting, Rome, July 2 - 8, 2000 